\title{
Postoperative chemotherapy for non-small cell lung cancer: A systematic review and meta-analysis
}

\author{
Artyom Sedrakyan, $\mathrm{MD}, \mathrm{PhD}^{\mathrm{a}, \mathrm{b}}$ \\ Jan van Der Meulen, $\mathrm{PhD}^{\mathrm{a}, \mathrm{b}}$ \\ Ken O'Byrne, MD \\ Joseph Prendiville, PhD, FRCP \\ Jennifer Hill, $\mathrm{PhD}^{\mathrm{e}}$ \\ Tom Treasure, MD, FRCS ${ }^{d}$
}

Background: Postoperative chemotherapy is currently not recommended for resected non-small cell lung cancer in many countries and centers. Recently, results of several large randomized clinical trials were reported with conflicting evidence. Accordingly, we sought to determine whether postoperative chemotherapy is associated with improved survival compared with that after surgical intervention alone.

Methods: Randomized clinical trials with cisplatin- or uracil plus ftorafur-containing regimens were included and evaluated separately. A systematic review that included randomized clinical trials performed before 1995 was identified and found to be of adequate quality. Further randomized controlled trials were identified by searching MEDLINE, EMBASE, and the Cochrane Controlled Trials Register from 1995 through 2004. In addition, the reference lists of articles and conference abstracts were searched. The logarithm of the hazard ratio and its standard error were calculated, and a fixed-effect model was used to combine the estimates.

Results: There were 7200 patients enrolled in 19 trials included in the analyses. An overall estimate of $13 \%$ relative reduction in mortality (95\% confidence interval, $7 \%-19 \%$ ) was found. There was $11 \%$ relative reduction in mortality associated with postoperative cisplatin (95\% confidence interval, $4 \%-18 \% ; P=.004$ ) and $17 \%$ associated with uracil plus ftorafur (95\% confidence interval, $5 \%-27 \% ; P=.006$ ) compared with that after surgical intervention alone. This means that there would be an additional survivor at 5 years for 25 patients treated with cisplatin or for 30 patients treated with uracil plus ftorafur.

Conclusions: Postoperative chemotherapy is associated with improved survival compared with that after surgical intervention alone. Selected patients with completely resected non-small cell lung cancer should be offered chemotherapy. the National Collaborating Centre for Acute Care, ${ }^{\mathrm{e}}$ Royal College of Surgeons of England, London, United Kingdom.

Received for publication March 16, 2004; revisions received April 15, 2004; accepted for publication April 23, 2004.

Address for reprints: Artyom Sedrakyan, $\mathrm{MD}, \mathrm{PhD}$, Clinical Effectiveness Unit, Royal College of Surgeons, 35-43 Lincoln's Inn Fields, London WC2A 3PE, United Kingdom (E-mail: asedrakyan@ rcseng.ac.uk).

J Thorac Cardiovasc Surg 2004;128:414-9

$0022-5223 / \$ 30.00$

Copyright $\odot 2004$ by The American Association for Thoracic Surgery

doi:10.1016/j.jtcvs.2004.04.027

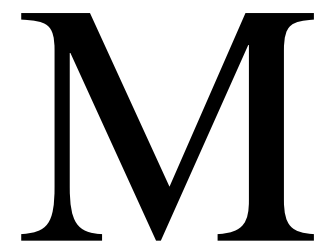

ore than 75,000 procedures in the United States and 3000 procedures in the United Kingdom are performed for early stage lung cancer, ${ }^{1,2}$ but even for patients with stage I and II non-small cell lung cancer (NSCLC), there is a death rate of $20 \%$ to $40 \%$ at 5 years with surgical intervention alone. ${ }^{3}$ The Non-small Cell Lung Cancer Collaborative Group (NSCLCCG) published a systematic review and meta-analysis in 1995, and a substantial section of the review was related to the issue of adjuvant chemotherapy use after surgical intervention. ${ }^{4,5}$ This section of this review, based on 12 randomized trials comparing adjuvant chemotherapy with surgical intervention alone, reported a tendency toward a reduction in mortality of $13 \%$ associated with cisplatin-based chemotherapy $(P=.08)$ and of $11 \%$ associated with uracil plus ftorafur (tefagur; UFT)-based chemotherapy $(P=.31)$. Because these results were 
not statistically significant, conventional wisdom is that postoperative chemotherapy should not be recommended after complete resection of early stage lung cancer, given the toxicity associated with the chemotherapy. ${ }^{6}$

Recently, the results of several large randomized clinical trials (RCTs) were reported, with conflicting evidence. Although the International Adjuvant Lung Cancer Trial $(\text { IALT })^{7}$ reported survival benefit associated with cisplatin chemotherapy, 2 other large trials, Adjuvant Lung Project Italy (ALPI) ${ }^{8}$ and the Big Lung Trial, ${ }^{9}$ did not report any survival advantage. If the effect of postoperative chemotherapy were true, given the large number of procedures conducted in the United Kingdom and the United States, a difference in survival that is perceived to be small (estimated at $4 \%-5 \%$ ) would mean that more than 3000 deaths in the United States and more than 150 deaths in the United Kingdom would be expected to be averted annually. Accordingly, we systematically searched for new evidence since the publication of the NSCLCCG review in 1995 and aimed to perform a meta-analysis of relevant trials to determine whether postoperative chemotherapy is associated with improved survival compared with that after surgical intervention alone. Because a small survival benefit represents a value judgment, we also sought to interpret the differences in survival from a clinical relevance perspective.

\section{Methods}

\section{Types of Studies and Participants}

The trials of mostly node-negative patients were included in this review. The trials of mostly node-positive patients include comparison of postoperative chemotherapy plus radiotherapy versus radiotherapy alone, are applicable to different group of patients, and should be analyzed separately. ${ }^{3}$ Additionally, node-positive patients compose the minority of patients undergoing surgical intervention for lung cancer, and there is some evidence that postoperative radiotherapy is detrimental for patients with stage I and II NSCLC. ${ }^{10}$ Thus node-positive trials require separate systematic review and are not included in this overview.

Only RCTs with cisplatin- or UFT-containing regimens were included in this overview. The trials of alkylating agents were not included because they were found to be harmful in the NSCLCCG review ${ }^{4}$ and are no longer used in current practice.

The trials of cisplatin- and UFT-containing regimens were evaluated in separate subgroup analyses. The following inclusion criteria were applied: (1) random allocation of study treatments, (2) use not combined with another experimental medication or procedure, and (3) postoperative use of chemotherapy.

For each trial, data on survival were collected in the intervention and control groups by one author (AS) and verified by all authors.

\section{Search Strategy for Identification of Studies}

The systematic review by NSCLCCG was found to be of adequate quality until 1995. All trials included in this review were included in the meta-analyses. New RCTs were identified by searching MEDLINE, EMBASE, and the Cochrane Controlled Trials Reg- ister from 1995 through 2004. A standard filter identifying RCTs was used for searching MEDLINE and EMBASE. These search algorithms are available at the Scottish Intercollegiate Guideline Network Web site (http://www.sign.ac.uk/methodology/filters. html). In addition, we searched the reference lists of RCTs, reviews, and conference reports to look for additional studies. Abstracts were included to minimize publication bias. ${ }^{11}$ Search of the databases, reference lists of articles, and conference reports identified 42 relevant abstracts related to postoperative chemotherapy. Seven unique trials met our inclusion criteria. ${ }^{7-9,12-15}$

\section{Statistical Analyses}

The logarithm of the hazard ratio (HR) and its SE were calculated from the original reports. ${ }^{16}$ Then a meta-analysis of the trials was performed stratified by each subgroup of medication (cisplatin and UFT). The test of homogeneity did not provide evidence for heterogeneity among the studies $\left(\chi^{2}\right.$ of $9.60, d f$ of 11 , and $P$ of .57 for cisplatin and $\chi^{2}$ of $10.86, d f$ of 6 , and $P$ of .09 for UFT). Therefore a fixed-effect model was used to combine the estimates. Additionally, cumulative meta-analyses were performed in which each trial's effect was added to the estimate observed in all previous studies. Finally, subgroup analyses of Japanese and European/North American participants in cisplatin trials were performed.

Funnel plot analyses were performed to determine publication bias in the trials. RevMan 4.2 was used in all analyses.

\section{Results}

The previous 12 trials of adjuvant chemotherapy included in the NSCLCCG review randomized a total of 1172 patients into the chemotherapy arm and 1140 patients into the control arm. ${ }^{4}$ In 7 new trials there were 2456 patients randomized to adjuvant chemotherapy and 2432 to surgical intervention alone. Altogether, there were 7200 patients enrolled in 19 trials that were included in the meta-analyses.

An overall estimate of $13 \%$ relative reduction in mortality (HR, 0.87; 95\% confidence interval [CI], 0.81-0.93; $P<$ .0001 ) associated with adjuvant chemotherapy was found. Meta-analyses by subgroups of cisplatin chemotherapy and UFT were performed separately. Analysis of funnel plots did not reveal the presence of substantial publication bias.

\section{Cisplatin-based Regimens}

Four new studies randomized a total of 1770 patients into a postoperative cisplatin-based regimen and 1748 patients into a control arm. Two studies ${ }^{7,14}$ reported a survival benefit, and 2 others $^{8,9}$ reported no survival advantage associated with postoperative chemotherapy (Figure 1). We have calculated the HR and 95\% CIs before and after combining the new studies with those included in the NSCLCCG report of 1995. The results before and after inclusion of the new studies are 0.87 (95\% CI, 0.74-1.02; $P=.08)$ and 0.89 (95\% CI, 0.82-0.96; $P=.003)$, with narrowed CIs and statistical significance (Figures 1 and 2). 


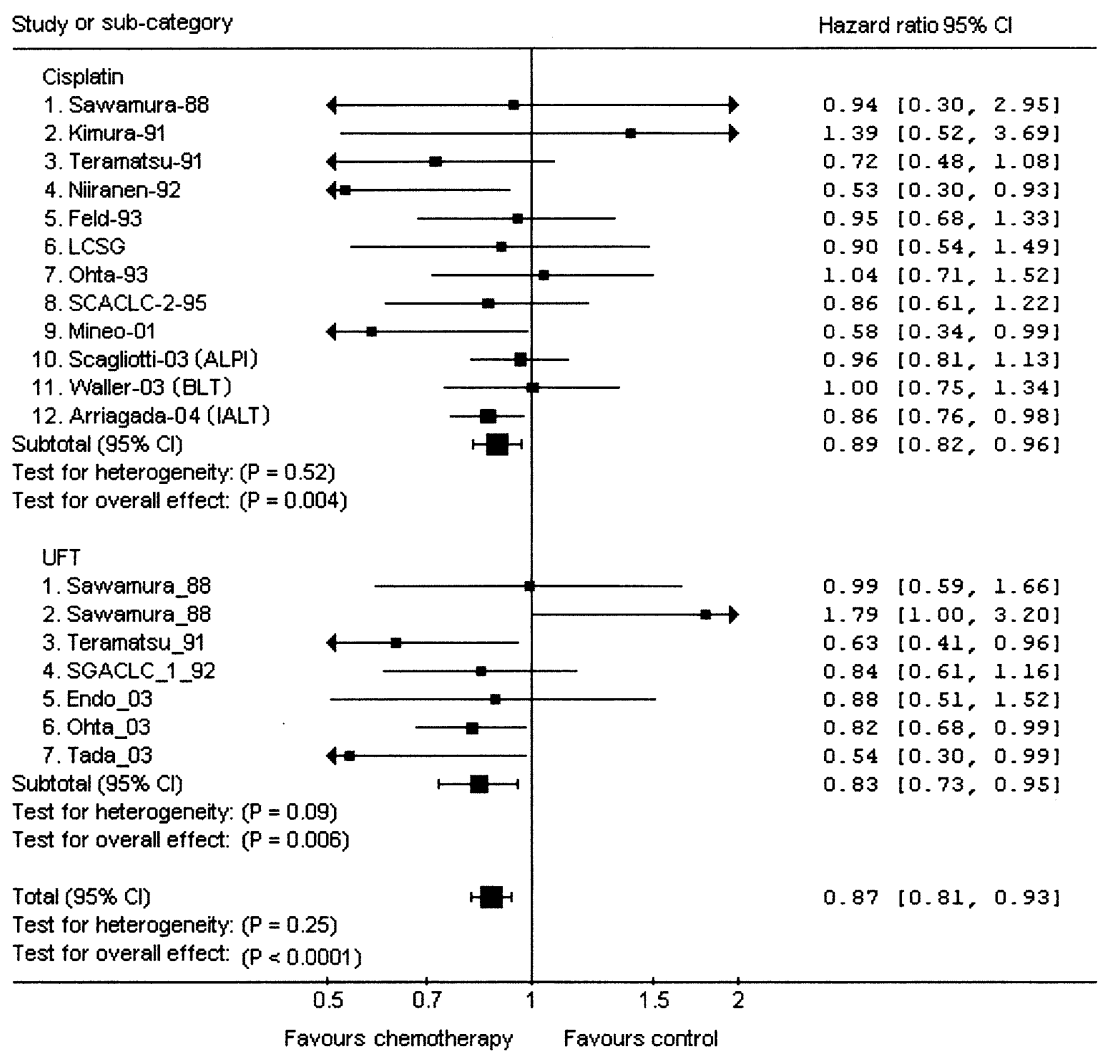

Figure 1. Meta-analysis of postoperative chemotherapy trials for NSCLC. The first 8 studies in the cisplatin subcategory and the first 4 studies in the UFT subcategory were originally combined by NSCLCCG. The horizontal axis is based on a log scale for HR (corresponds to relative risk) ranging from 0.5 to 2.0. The black boxes and lines correspond to HR estimates and $95 \% \mathrm{Cls}$ for individual studies. Arrows show that the $95 \% \mathrm{Cl}$ extends beyond 0.5 or 2.0 .

Subgroup analysis of Japanese versus European/North American trials has shown no evidence for a survival difference between participants in these countries (Figure $3)$.

\section{UFT-based Regimens}

Three new studies randomized 686 patients into the UFT arm and 684 patients into a control arm (surgical intervention alone). Two reports from one study ${ }^{12,17}$ and another separate investigation ${ }^{15}$ reported a statistically significant survival advantage, whereas the third study showed a trend toward improved survival associated with UFT (Figure 1). ${ }^{13}$ After combining the estimates from these studies with those included in the NSCLCCG report, the HR has changed from 0.89 (95\% CI, 0.72-1.10) to 0.83 (95\% CI, 0.73-0.95; $P=$ .006 ; Figures 1 and 2). Sensitivity analyses involving exclusion of two 15-year-old studies (Figure 1, UFT section, Sawamura_88 and Sawamura_88) have shown an even larger reduction in mortality with UFT-based therapy (HR, 0.78 ; 95\% CI, 0.68-0.90; $P=.0007$ ).

\section{Discussion}

In this study we found that postoperative chemotherapy with both cisplatin- and UFT-based regimens is associated with survival benefits compared with that after surgical intervention alone for NSCLC. We found an $11 \%$ relative reduction in mortality associated with cisplatin and a $17 \%$ relative reduction associated with UFT. The cisplatin-based trials enrolled patients with stage I to IIIA disease (mostly I-II). ${ }^{3,7-9}$ Survival in the control group (surgical intervention alone) was approximately $45 \%$ at 5 years. As a result, the $11 \%$ relative reduction in mortality translates into an approximately $4 \%$ absolute improvement in survival. Thus, it would be necessary to treat approximately 25 patients to prevent one additional death at 5 years. In contrast, UFTbased regimens were mostly used for stage I disease, with few patients having stage II disease. ${ }^{12,13,15}$ Survival in the control group (surgical intervention alone) was approximately $80 \%$ at 5 years. Accordingly, a 17\% relative reduction in mortality transforms into an approximately $3.5 \%$ absolute improvement in survival at 5 years. Thus, it would 


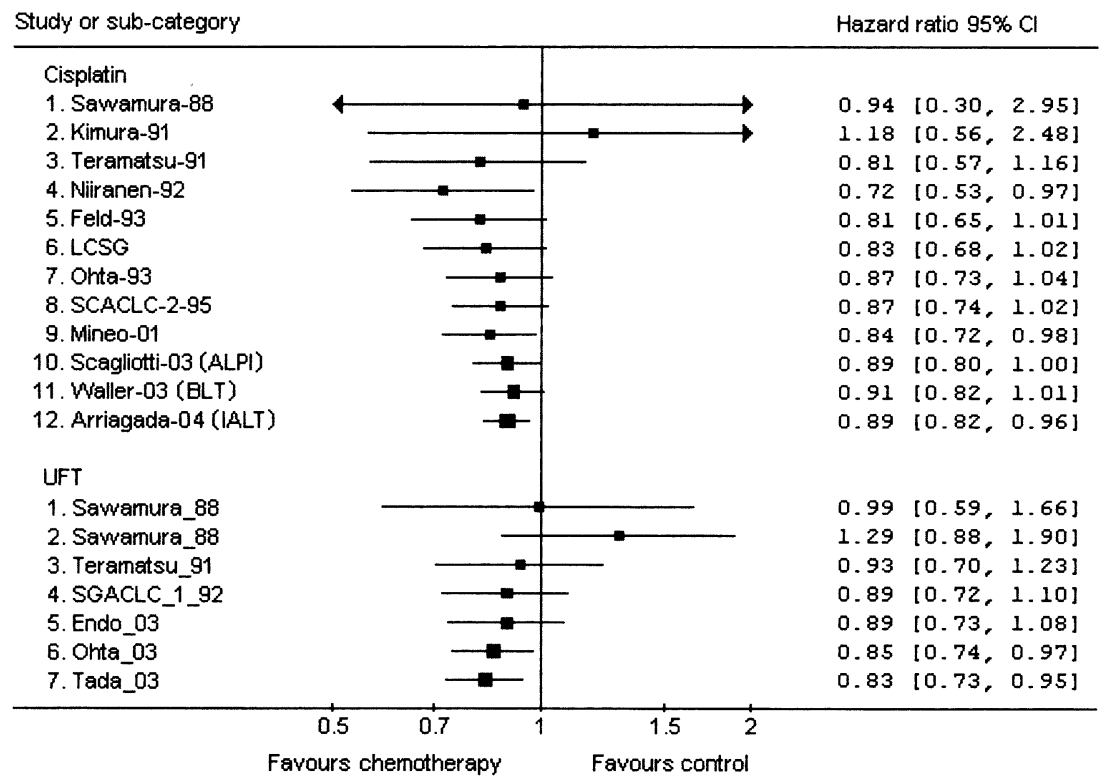

Figure 2. Cumulative meta-analysis of postoperative chemotherapy trials for NSCLC. Studies are ordered according to the date of the first report. The first 8 studies in the cisplatin subcategory and the first 4 studies in the UFT subcategory were originally combined by NSCLCCG. The horizontal axis is based on a log scale for HR (corresponds to relative risk) ranging from 0.5 to 2.0 . The black boxes and lines correspond to HR estimates and 95\% Cls for individual studies. Arrows show that the $95 \% \mathrm{Cl}$ extends beyond 0.5 or 2.0 . The estimate of each individual trial is added to the estimate observed in all previous studies to perform cumulative meta-analyses. If the direction of the association is the same, then no difference in the overall estimate is observed, but $95 \%$ Cls shrink because of increased sample size.

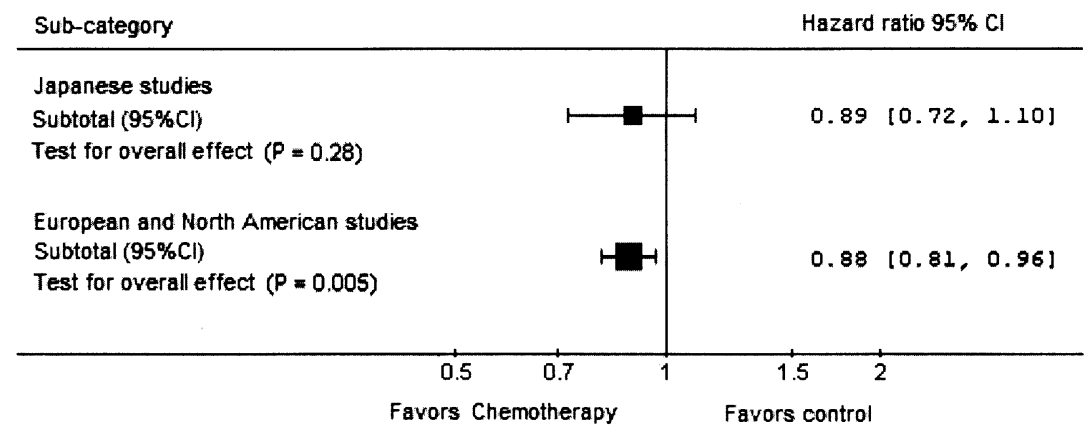

Figure 3. Subgroup analyses of cisplatin-based postoperative chemotherapy trials by Japanese and European/ North American participants.

take about 30 treated patients to prevent one additional death at 5 years.

All UFT trials have been conducted in Japan, whereas cisplatin trials were performed in a number of countries, including Japan, Europe, and North America. In subgroup analyses we found no evidence for a survival difference between Japanese and European/North American participants in cisplatin trials (Figure 3), and therefore there is no a priori reason to reject the applicability of UFT findings to Europe and North America. Because UFT is not yet avail- able in North America and Europe, it might be important to invigorate previous efforts to initiate RCTs and to investigate the applicability of these encouraging findings.

One limitation of our study was that we did not perform formal quality evaluation for the studies included in the overview; however, most included studies were large multicenter trials, and their quality is hardly questionable. One other limitation might be publication bias (negative studies not being reported). However, funnel plots were not indicative of substantial publication bias, and our sensitivity 
analyses determined that even if there were studies enrolling more than 1000 patients that would show a statistically significant $20 \%$ higher risk of mortality for cisplatin chemotherapy (which is very unlikely), the overall estimate would still favor cisplatin chemotherapy and would be statistically significant.

The results of the postoperative chemotherapy studies are supported by those of published studies of preoperative and perioperative chemotherapy that likewise demonstrate a benefit in survival. ${ }^{18-20}$ The largest of these ${ }^{20}$ randomized 355 eligible patients to induction and postoperative chemotherapy versus surgical intervention alone. Although survival differences did not achieve overall significance in this study, there was an $8.6 \%$ absolute reduction in mortality in the chemotherapy treatment arm after 4 years.

Previous guidelines were heavily influenced by the finding that the effect of postoperative chemotherapy was not statistically significant $(P=.08$ for cisplatin and $P=.31$ for UFT), and survival benefit at 5 years for cisplatin was considered small in the light of possible toxicity. ${ }^{6}$ After the IALT study results were reported, most centers in North America started to recommend at least chemotherapy consultation for all patients with stage IB to IIIA disease. However, this evidence did not receive as much attention in the United Kingdom and many European countries. There was concern that IALT is only one individual study that has shown this benefit. Our meta-analysis determined that the evidence of survival advantage was available before the IALT results were published and does not change even after adding the results of other recent large trials. Meta-analysis has an advantage of pulling many underpowered clinical trials to determine that survival advantage of $4 \%$ is not by chance alone.

It is also important to assert that a small increase in survival is common to many oncologic strategies, and a benefit of this size is considered acceptable for the prescription of adjuvant chemotherapy in colorectal and breast cancers. Moreover, there is some evidence to suggest that the magnitude of the benefit associated with postoperative chemotherapy might be improved in the future. For example, the cisplatin and etoposide or vindesine or vinblastine regimens used in a majority of patients in these studies are inferior to regimens such as mitomycin, ifosfamide, and cisplatin or mitomycin, vinblastine, and cisplatin and the cisplatin doublet regimens incorporating newer cytotoxic agents, which are currently used as a standard therapy in metastatic disease. ${ }^{21-23}$

The effectiveness of UFT-based regimens has not been discussed previously because the estimate of the absolute survival difference was even smaller than the estimate for cisplatin-based chemotherapy, and the difference was much more likely to occur by chance alone $(P=.31)$. Because UFT-based regimens are generally less toxic than cisplatin- based regimens, with severe toxicity occurring in less than $3 \%$ of patients, ${ }^{3,12,13}$ further research should elucidate whether UFT-based regimens might be considered as alternatives to cisplatin and as treatments of choice for postoperative chemotherapy, particularly for stage I disease. The mode of action of long-term UFT therapy might be different from that of a standard cytotoxic agent. ${ }^{24}$ This raises the possibility that cisplatin-based chemotherapy followed by maintenance UFT might optimize outcomes for patients with resected NSCLC.

Mostly stage I to II, otherwise healthy patients with good performance status (Karnofsky, >80; Eastern Cooperative Oncology Group, $<2$ ) were enrolled in the trials of postoperative chemotherapy. In addition, some patients received adjuvant radiotherapy in these trials, and its effect on survival requires further evaluation because there is some evidence that postoperative radiotherapy is detrimental for stage I and II NSCLC but might reduce local relapse for stage IIIA disease. ${ }^{10}$

\section{Conclusions}

Selected patients with completely resected NSCLC should be offered postoperative chemotherapy. This would influence the current practice of postoperative management after a major lung resection procedure. Further research should elucidate patient preferences regarding the tradeoff between improved life expectancy and toxicity and establish the role of UFT and other oral fluoropyrimidines in the management of resected NSCLC.

We thank Veena Paes, Louise Thomas, and Rachel Southon for their work on the initial searches, identifying the hard copies of the studies, and other logistical support.

\section{References}

1. Agency for Health Care Research and Quality HCCaUP. Available at: http://hcup.ahrq.gov/HCUPNet.asp. Accessed August 2003.

2. Goldstraw P. The expansion of consultant provision for thoracic surgery in the UK. Available at: http://www.scts.org. Accessed 2003.

3. Detterbeck FC, Rivera MP, Socinski MA, Rosenman JG. Diagnosis and treatment of lung cancer: an evidence-based guide for the practicing clinician. Philadelphia: W. B. Saunders; 2001.

4. Non-small Cell Lung Cancer Collaborative Group. Chemotherapy in non-small cell lung cancer: a meta-analysis using updated data on individual patients from 52 randomised clinical trials. BMJ. 1995; 311(7010):899-909.

5. Non-small Cell Lung Cancer Collaborative Group. Chemotherapy for non-small cell lung cancer (Cochrane Review). In: The Cochrane Library, Issue 2. Chichester (UK): John Wiley \& Sons, Ltd; 2003.

6. Logan DM, Lochrin CA, Darling G, Eady A, Newman TE, Evans WK. Adjuvant radiotherapy and chemotherapy for stage II or IIIA nonsmall-cell lung cancer after complete resection. Provincial Lung Cancer Disease Site Group. Cancer Prev Control. 1997;1(5):366-78.

7. Arriagada R, Bergman B, Dunant A, et al. Cisplatin-based adjuvant chemotherapy in patients with completely resected non-small cell lung cancer. $N$ Engl J Med. 2004;350(4):351-60.

8. Scagliotti GV, Fossati R, Torri V, et al. Randomized study of adjuvant chemotherapy for completely resected stage I, II, or IIIA non-smallcell Lung cancer. J Natl Cancer Inst. 2003;95(19):1453-61.

9. Waller D, Fairlamb DJ, Gower N, et al. The Big Lung Trial (BLT): 
determining the value of cisplatin-based chemotherapy for all patients with non-small cell lung cancer (NSCLC). Preliminary results in the surgical setting. Lung Cancer. 2003;41(suppl 2):S54.

10. PORT Meta-analysis Trialist Group. Postoperative radiotherapy in non-small-cell lung cancer: systematic review and meta-analysis of individual patient data from nine randomized controlled trials. Lancet. 1998;25(352):257-63.

11. Egger M, Smith GD, Altman D. Systematic reviews in health care, meta analysis in context. London, United Kingdom: BMJ Publishing Group; 2001.

12. Ohta M, Kato H, Hata E, et al. A randomised phase III trial of adjuvant chemotherapy with UFT for completely resected pathological stage I (T1NOM0,T2NOM0) adenocarcinoma of the lung [abstract]. Lung Cancer. 2003;41(Suppl 2):S3.

13. Endo C, Saito Y, Iwanami H, et al. A randomized trial of postoperative UFT therapy in p stage I, II non-small cell lung cancer: North-east Japan Study Group for Lung Cancer Surgery. Lung Cancer. 2003; 40(2):181-6.

14. Mineo TC, Ambrogi V, Corsaro V, Roselli M. Postoperative adjuvant therapy for stage IB non-small-cell lung cancer. Eur J Cardiothorac Surg. 2001;20(2):378-84.

15. Tada H, Yasumitsu T, Luchi K, Taki T, Kodama K, Matsumura A. Randomised control study of adjuvant chemotherapy with UFT alone or plus cisplatin and vindesine in patients with completely resected non-small cell lung cancer. Lung Cancer. 2003;41(suppl 2):S54.

16. Parmar MK, Torri V, Stewart L. Extracting summary statistics to perform meta-analyses of the published literature for survival endpoints. Stat Med. 1998;17(24):2815-34.

17. Kato H, Tsuboi M, Ohta M, et al. A randomized phase III trial of adjuvant chemotherapy with UFT for completely resected pathological stage I (T1N0M0, T2NOM0) adenocarcinoma of the lung [abstract]. Prog Proc Am Soc Clin Oncol. 2003;22:621.

18. Rosell R, Gomez-Codina J, Camps C, et al. A randomized trial comparing preoperative chemotherapy plus surgery with surgery alone in patients with non-small-cell lung cancer. N Engl J Med. 1994; 330(3):153-8.

19. Roth JA, Atkison EN, Fossella F, et al. Long-term follow up of patients enrolled in a randomized trial comparing perioperative chemotherapy and surgery with surgery alone in resectable stage IIIa non-small-cell lung cancer. Lung Cancer. 1998;21(1):1-6.

20. Depierre A, Milleron B, Moro-Sibilot D, et al. Preoperative chemotherapy followed by surgery compared with primary surgery in resectable stage I(except T1N0), II, and IIIa non-small-cell lung cancer. J Clin Oncol. 2002;20(1):247-53.

21. Crino L, Clerici M, Figoli F, et al. Chemotherapy of advanced nonsmall-cell lung cancer: a comparison of three active regimens. A randomized trial of the Italian Oncology Group for Clinical Research (G.O.I.R.C.). Ann Oncol. 1995;6(4):347-53.

22. Bonomi P, Kim K, Fairclough D, et al. Comparison of survival and quality of life in advanced non-small-cell lung cancer patients treated with two dose levels of paclitaxel combined with cisplatin versus etoposide with cisplatin: results of an Eastern Cooperative Oncology Group trial. J Clin Oncol. 2000;18(3):623-31.

23. Schiller JH, Harrington D, Belani CP, et al. Comparison of four chemotherapy regimens for advanced non-small-cell lung cancer. N Engl J Med. 2002;346(2):92-8.

24. Basaki Y, Chikahisa L, Aoyagi K, et al. Gamma-Hydroxybutiyric acid 5-fluorouracil, metabolites of UFT, inhibit angiogenesis induced by vascular endothelial growth factor. Angiogenesis. 2001;4(3):163-73.

Access to The Journal of Thoracic and Cardiovascular Surgery Online is reserved for print subscribers!

Full-text access to The Journal of Thoracic and Cardiovascular Surgery Online is available for all print subscribers. To activate your individual online subscription, please visit The Journal of Thoracic and Cardiovascular Surgery Online, point your browser to http://www.mosby.com/jtcvs, follow the prompts to activate your online access, and follow the instructions. To activate your account, you will need your subscriber account number, which you can find on your mailing label (note: the number of digits in your subscriber account number varies from 6 to 10). See the example below in which the subscriber account number has been circled:

\section{Sample mailing label}

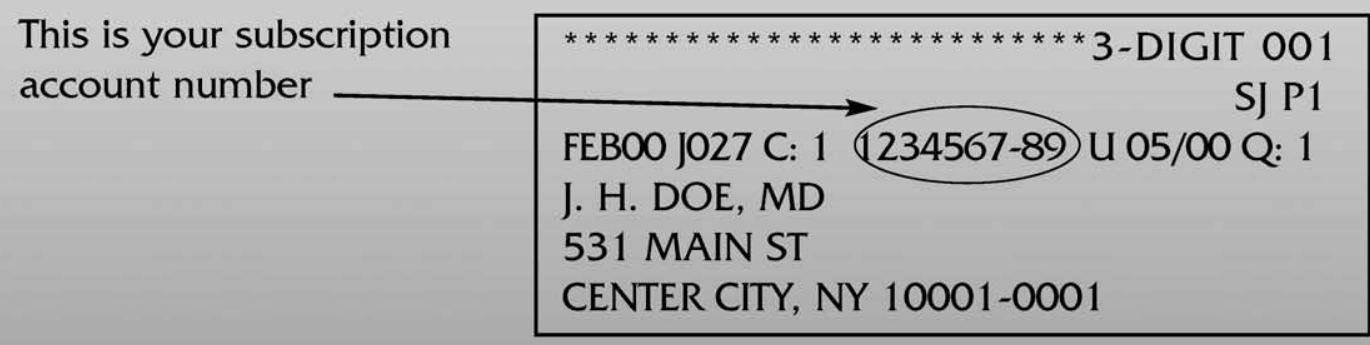

Personal subscriptions to The Journal of Thoracic and Cardiovascular Surgery Online are for individual use only and may not be transferred. Use of The Journal of Thoracic and Cardiovascular Surgery Online is subject to agreement to the terms and conditions as indicated online. 